\title{
Lucas-Interpretation on Isabelle's Functions
}

\author{
Walther Neuper \\ Johannes Kepler University, Linz, Austria \\ walther.neuper@jku.at
}

\begin{abstract}
Software tools of Automated Reasoning are too sophisticated for general use in mathematics education and respective reasoning, while Lucas-Interpretation provides a general concept for integrating such tools into educational software with the purpose to reliably and flexibly check formal input of students.

This paper gives the first technically concise description of Lucas-Interpretation at the occasion of migrating a prototype implementation to the function package of the proof assistant Isabelle. The description shows straightforward adaptations of Isabelle's programming language and shows, how simple migration of the interpreter was, since the design (before the function package has been introduced to Isabelle) recognised appropriateness of Isabelle's terms as middle end.

The paper gives links into the code in an open repository as invitation to readers for re-using the prototyped code or adopt the general concept. And since the prototype has been designed before the function package was implemented, the paper is an opportunity for recording lessons learned from Isabelle's development of code structure.
\end{abstract}

\section{Introduction}

This paper concerns application of Automated Reasoning (AR) to education, the issue to narrow the gap between powerful, but highly sophisticated technologies of AR on the one side and requirements of education in mathematics as taught in engineering studies. Lucas-Interpretation (in the sequel abbreviated by $L I$ ) provides a general concept for integrating AR tools into educational software with the purpose to reliably and flexibly check formal input of students.

A prerequisite for $L I$ is a logical framework, which models rigorous formal derivation, in particular forward reasoning. There are several educational software products of this kind: [4] calls "structuredderivation" what here is called "calculation" and uses the PVS prover in the background, IMPS is an Interactive Mathematical Proof System intended to provide organizational and computational support for the traditional techniques of mathematical reasoning [11], and in geometry software AR tools are used [5, 15], too. But all the above mentioned systems are poor in guided interaction.

A logic course for freshmen in AI and Computer Science ${ }^{1}$ accompanies all content with software. And corresponding experience shows [ [8] again, that much of the software lacks interactivity and feedback sufficient for independent learning ${ }^{2}$ in particular in application to mathematics.

In principle [32], AR provides the most powerful tools for independent learning in formal mathematics, captured by naive requirements like these: given a formal problem specification, AR checks steps of forward reasoning towards a problem solution, a step determined by an input term or by an input theorem to be applied to the current state of solution; and the system can provide a next step, if a student gets stuck. Nowadays such naive requirements might be reconsidered, since proof assistants like Isabelle [37] hide the intricacies of AR tools and present notation to the user close to standard mathematics.

\footnotetext{
${ }^{1}$ http://fmv.jku.at/logic/

${ }^{2}$ Currently independent learning is best addressed by "flipped classes" [40]
}

J. Marcos, W. Neuper and P. Quaresma (Eds.): Theorem Proving Components for Educational Software 2020 (ThEdu'20)

EPTCS 328, 2020, pp. 79-95 doi 10.4204/EPTCS.328.5 
$L I$ aims at meeting these requirements for more than a decade, when Peter Lucas 3 shifted his interests from programming languages [24] to education. His specific contribution has been named after him, and now reveals the ingenuity of the original design, when the prototype has been migrated: a proprietary version [22] was migrated to Isabelle's function package [20, 21] with surprising little effort.

Herewith the first technically concise description of $L I$ is given, after theoretical considerations [27] and application oriented discussions [28, 29, 30]. Since $L I$ was implemented in the $I S A C$-project ${ }^{4}$ several years before the function package was introduced to the proof assistant Isabelle, we take the paper as an opportunity for recording lessons learned from Isabelle's development.

The paper is structured as follows: $\$ 2$ introduces $L I$, the concept in $\$ 2.1$ illustrated with examples in $\$ 2.2$. $\$ 2.3$ notes $L I$ 's relevance for educational mathematics software. Adaptation of the programs for $L I$ to Isabelle's function package is described in $\$ 3$, where $\$ 3.1$ describes the tactics, $\$ 3.2$ the tacticals and $\$ 3.3$ briefly describes evaluation by rewriting. The parse-tree generated by the function package is interpreted by $L I$ as shown in $\$ 4 \$ 4.1$ gives a description of how the parse-tree is scanned, $\$ 4.2$ of how LI uses Isabelle's Proof.context and $\$ 4.3$ of how the prototype's mathematics-engine embeds and guards $L I$. Lessons have been learned from Isabelle's code structure and development process \$5.1, from specific Isabelle features $(\$ 5.2)$ and now direct future prototyping $(\$ 5.3)$. In $\$ 6$ the summary concludes with expectations on $L I$ widely applied to education in mathematics.

\section{Lucas-Interpretation $(\boldsymbol{L I})$}

The interpreter is named after the inventor of top-down-parsing in the ALGOL project [24], Peter Lucas. As a dedicated expert in programming languages he initially objected "yet another programming language" (in analogy to "Yacc" [16]), but then he helped to clarify the unusual requirements for a novel programming language in the $I S A C$-project, which later led to the notion of $L I$.

$L I$ is the most prominent component in a prototype developed in the ISAC-project, there embedded in a mathematics-engine, which interacts with a dialogue-module in a Java-based front-end managing interaction with students (briefly touched below in $\$ 2.3$.

\subsection{The Concept of $L I$}

The concept of LI is simple: LI acts as a debugger on functional programs with hard-coded breakpoints, where control is handed over to a student; a student, however, does not interact with the software presenting the program, but with a software presenting steps of forward reasoning, where the steps are rigorously constructed by tactics acting as the break-points mentioned. Following the LI terminology, we will call "programs" the functions defined with the function package and refer to their evaluation as "execution".

Types occurring in the signatures of $L I$ are as follows. Besides the program of type Program. $\mathrm{T}$ there is an interpreter state Istate.T, a record type passing data from one step of execution to the next step, in particular a location in the program, where the next step will be read off, and an environment for evaluating the step. As invisible in the program language as the interpreter state is Calc. T, a "calculation"

\footnotetext{
$\sqrt[3]{\text { https://austria-forum.org/af/AustriaWiki/Peter_Lucas_(Informatiker) }}$

4 https://isac.miraheze.org/wiki/History
} 
as a sequence of steps in forward reasoning, a variant of "structured derivations" [3]. Visible in the language and in the signature, however, are the tactics Tactic. T, which create steps in a calculation.

Novel in connection with calculations is the idea to maintain a logical context in order to provide automated provers with data required for checking input by the student. Isabelle's Proof .context, as is, turned out perfect for this task [23].

The signatures of the main functions, the functions find_next_step, locate_input_tactic and locate_input_term, are as follows:

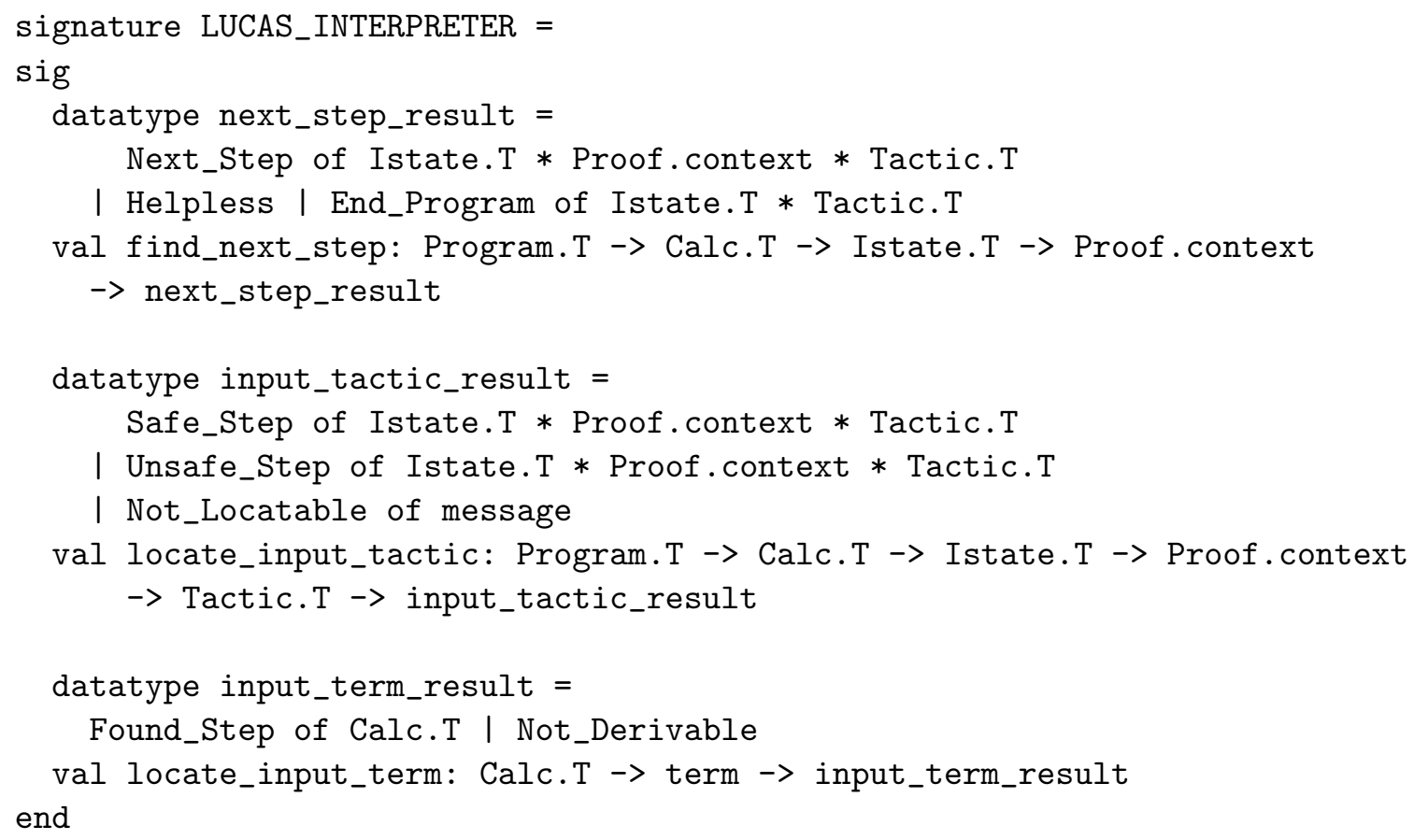

find_next_step accomplishes what usually is expected from a Program.T: find a Next_Step to be executed, in the case of $L I$ to be inserted into a Calc.T under construction. This step can be a Tactic.T, directly found in next_step_result, or a term produced by applying the tactic. If such a step cannot be found (due to previous student interaction), $L I$ is Helpless.

locate_input_tactic gets a Tactic.T as argument (which has been input and checked applicable in Calc.T) and tries to locate it in the Prog.T such that a Next_Step can be generated from the subsequent location in the Program. T. A step can be an Unsafe_Step, if the input Tactic. T cannot safely be associated with any tactic in the Program.T. This function has a signature similar to find_next_step (here the respective input Tactic.T and the one in the result are the same) in order to unify internal technicalities of $L I$.

locate_input_term tries to find a derivation from the current Proof.context for the term input to Calc.T and to locate a Tactic.T in the Program.T, which as Found_Step allows to find a next step later. Such a Found_Step can be located in another program (a sub-program or a calling program); thus Program.T, Istate.T and Proof . context are packed into Calc.T at appropriate positions and do not show up in the signature.

AR is concern of the third function locate_input_term, actually a typical application case for Isabelle's Sledgehammer but not yet realised and preliminarily substituted by ISAC's simplifier. locate_input_term is the function used predominantly in interaction with students: these input term by term into 
the calculation under construction (as familiar from paper\&pencil work) and $L I$ checks for correctness automatically.

\subsection{Examples for $L I$}

$L I$ 's novel concept relating program execution with construction of calculations is best demonstrated by examples. The first one is from structural engineering given by the following problem statement:

Determine the bending line of a beam of length $L$, which consists of homogeneous material, which is clamped on one side and which is under constant line load $q_{0}$; see the Figure right.

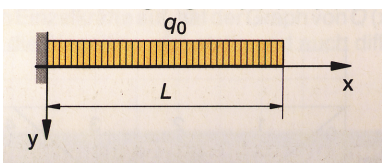

This problem is solved by the above program, which is shown by a screen-sho ${ }^{5}$ This problem is solved by the above program, which is shown by a screen-sho ${ }^{6}$ in order to demonstrate the colouring helpful for programmers (compare the old bare string version in [22, p. 92]). The program is implemented

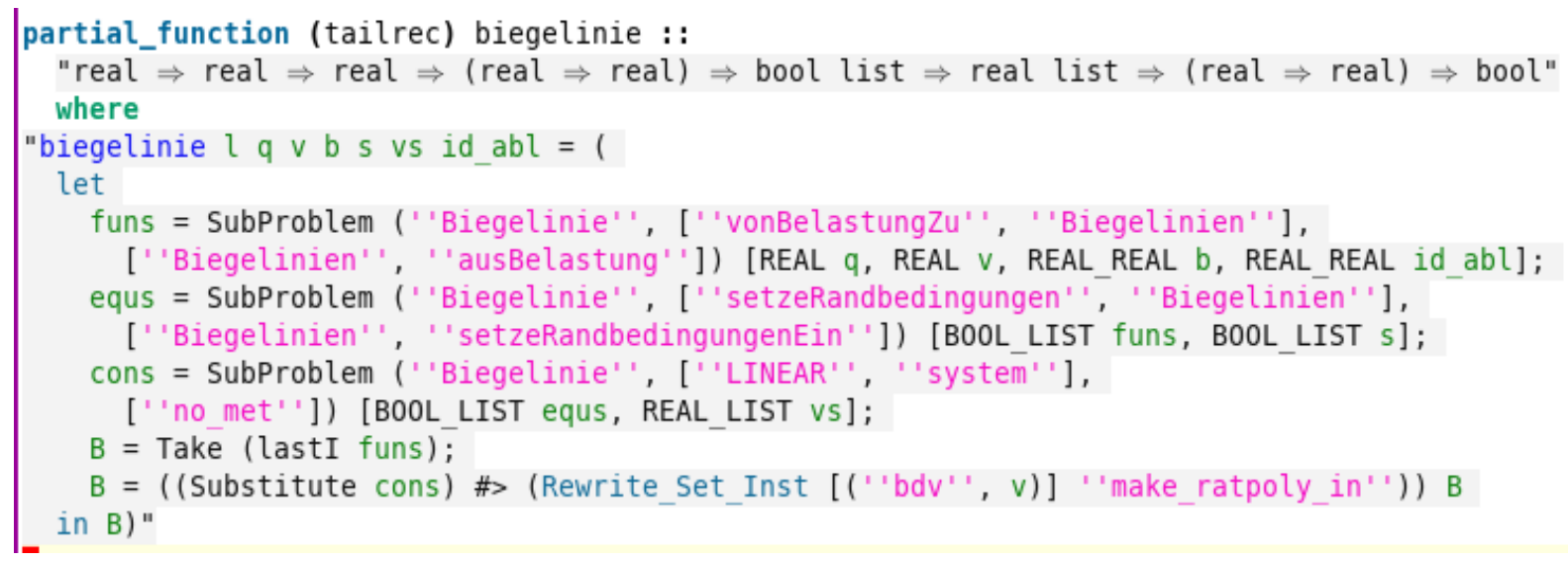

Figure 1: The program implemented by the function package

as a partial_function: it calculates results for a whole class of problems and termination cannot be proven in such generality, although preconditions guard execution. The program reflects a "divide \& conquer" strategy by calling three SubProblems. It was implemented for field-tests in German-speaking countries, so arguments of the SubProblems are in German, because they appear in the calculation as well. The third SubProblem adopts the format from Computer Algebra. The calculation, if finished successfully, looks as follows (a screen-shot of intermediate steps on the prototype's front-end is shown in [22, p. 97]):

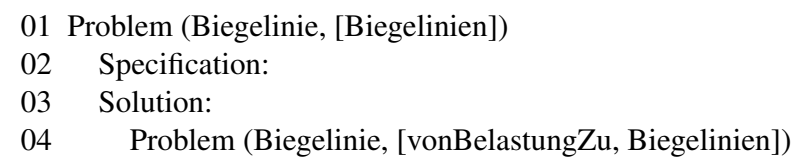

\footnotetext{
${ }^{5}$ The corresponding code is at https://hg.risc.uni-linz.ac.at/wneuper/isa/file/df1b56b0d2a2/src/ Tools/isac/Knowledge/Biegelinie.thy\#l174

${ }^{0}$ The corresponding code is at https://hg.risc.uni-linz.ac.at/wneuper/isa/file/df1b56b0d2a2/src/ Tools/isac/Knowledge/Biegelinie.thy\#l174
} 


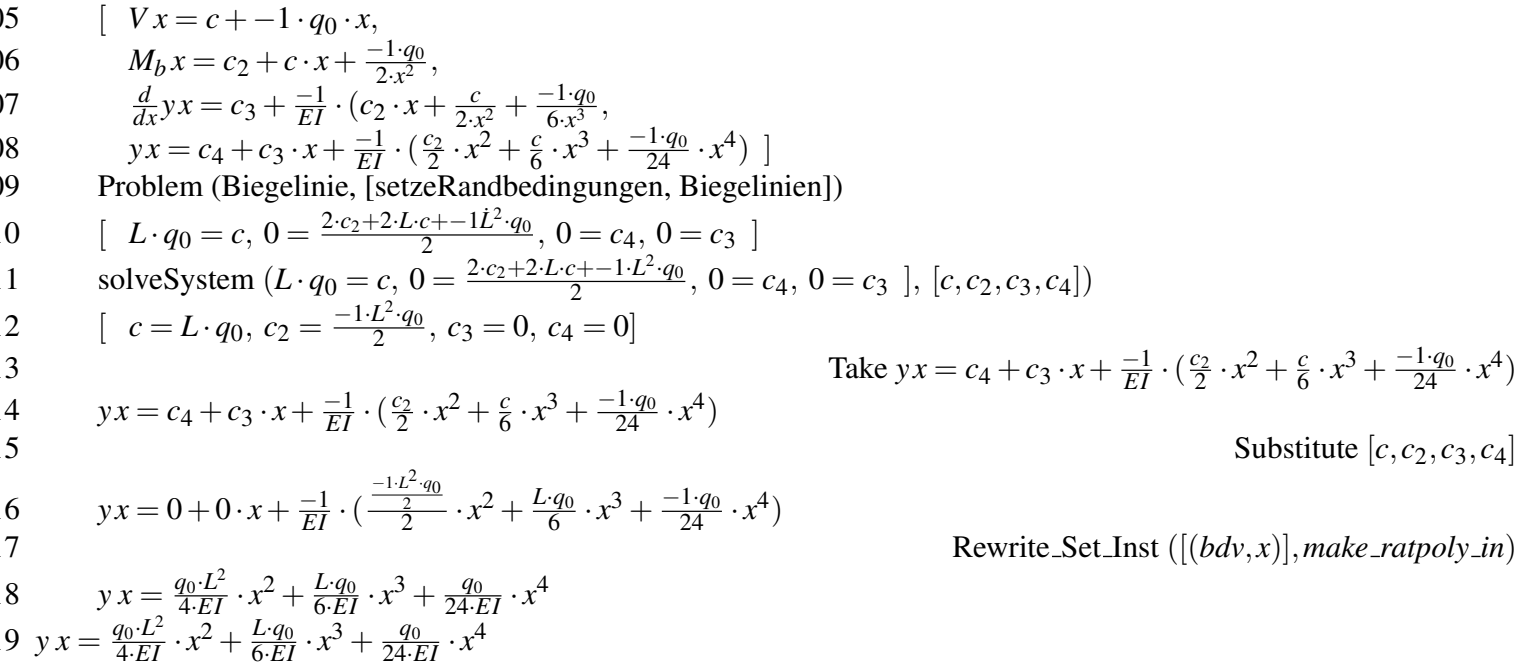

The calculation is what a student interacts with. Above the Specification is folded in, the specification phase and respective user interaction is skipped here, because user-interaction in this phase is not guided by $L I$ (while the result of the phase, a concise formal specification of the problem is a necessary pre-condition for $L I$ ). The Solution must be constructed step-wise line by line (while the line numbers do not belong to the calculation) in forward reasoning. A student inputs either (1) a term (displayed on the left above with indentations) or (2) a tactic (shifted to the right margin). If the student gets stuck (3) a next step (as a term or a tactic) is suggested by $L I$, which works behind the scenes; the corresponding functions have been introduced in $\$ 2.1$ (for (1) locate_input_term, (2) locate_input_tactic and for (3) find_next_step). A student can combine actions (1)..(3) arbitrarily.

This example hopefully clarifies the relation between program and calculation in LI: execution of the program stops at tactics (above called break-points), displays the tactic or the result produced by the tactic (as decided by the dialogue-module) in the calculation under construction, hands over control to the student working on the calculation and resumes checking user input.

A typical example from Computer Algebra shall shed light on a notions introduced later, on tacticals. The program in Fig 2 simplifies rational terms (typed as "real" due to limited development resources):

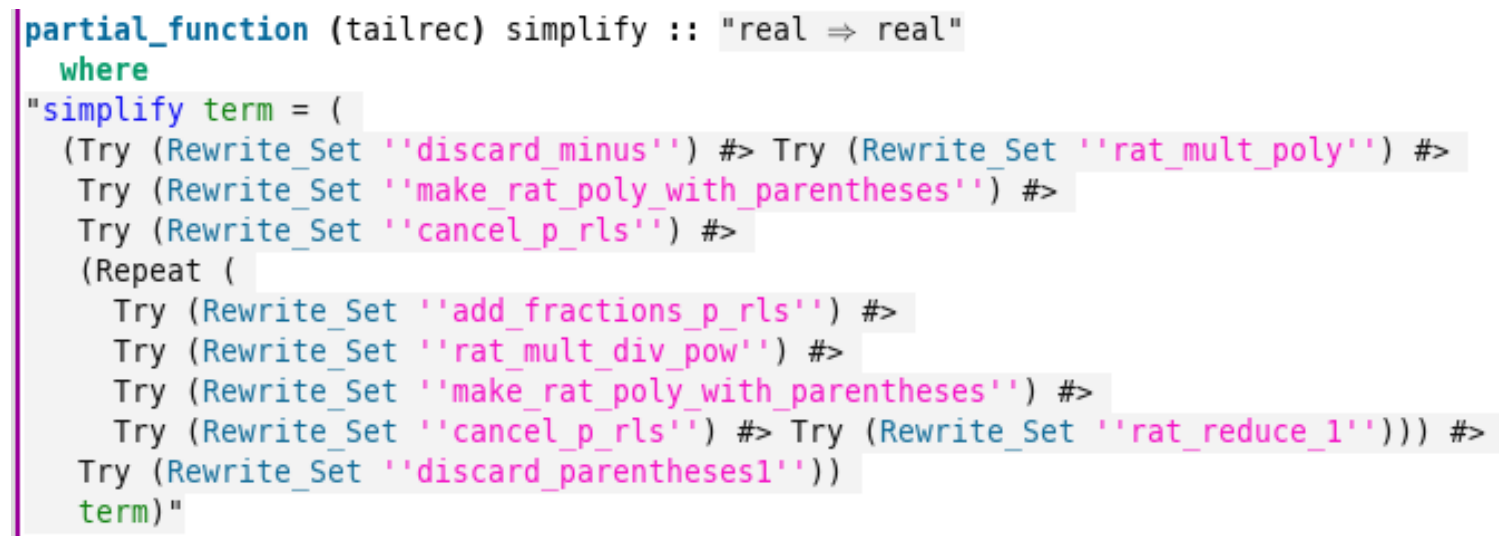

Figure 2: Simplification implemented by the function package 
The program executes the tactic Rewrite' _Set ${ }^{7}$ with different canonical term rewriting systems (called "rule sets" in ISAC) guided by tacticals. Chained functions (by \#>) are applied curried to term. The initial part of the chain, from ' 'discard_minus' ' to ' 'cancel' ' does preprocessing, for instance replaces $a-b$ by $a+(-b)$ in ' 'discard_minus' ' for reducing the number of theorems to be used in simplification. The second chain of Rewrite_Set is Repeated until no further rewrites are possible; this is necessary in case of nested fractions.

In cases like the one above, a confluent and terminating term rewrite system, not only all correct input terms are accepted, but also all incorrect input is rejected as Not_Derivable by the function locate_input_term.

\subsection{LI's Relevance for Educational Mathematics Software}

Main concern of this paper is describing technical aspects of $L I$. But here is a brief summary of promises for education, which should make the paper selfcontained for educators - promises derived from $L I$ 's three functions introduced on $\mathrm{p} 81$ and interpreted with respect to students' interaction on $\mathrm{p} 83$.

$\boldsymbol{L I}$ is generally usable by all software, which constructs a solution for a given problem - this, in principle, includes all programs, for instance, written in Mathematica 8 and millions of educational software products: just (re-)write the programs calculating a solution as shown in Fig 1. Doing so would cover most of mathematics as taught at engineering faculties as well as taught at high-schools.

Of course, that is an endless deal of work: but it can be done by lecturers and teachers, who like writing software for the problems they teach. And, of course, in order to make their development efficient, comprehensive libraries need to be available free of charge (like some of the libraries available for Mathematica). Such libraries would be concern of publicly funded academic development projects.

Such projects can build upon mechanised mathematics knowledge, which goes far beyond the contents of undergraduate studies (for instance in Isabelle's archive of formal proofs [1]) in mathematics and in engineering studies. From a certain point, development would become efficient and sustainable, because based on software with a huge user community extending the communities working on mechanisation of mathematics knowledge.

$\boldsymbol{L I}$ features "models of mathematics", transparent, complete and interactive models, as already described in [32] and briefly repeated here. Mathematical problem solutions (in the sequel called calculations) can be "transparent" by mouse-click on an element of a calculations, which immediately leads to the respective definition 9 and related properties together with (more or less) readable proofs. Thus mathematical calculations can be "complete" with respect to deduction from first principles (where students can follow these deductions at their own pace, or even not). And mathematical calculations can be "interactive" relying on $L I$ as described in this paper.

What makes such calculations to "models of mathematics" is the fact, that it covers all of mathematics which can be mechanised, while most computer mathematicians agree that (almost all of) mathematics can be mechanised (as soon as it is fully understood). And each item of knowledge and each step in a calculations is proven correct, unambiguously and based on logic - which fullfills the main property

\footnotetext{
${ }^{7}$ Isabelle's document preparation system preserves the apostroph from the definition, while Isabelle's pretty printer drops it in the presentation of the program as above. The apostroph excapes the underscore as required by Isabelle's lexer.

${ }^{8}$ https://www.wolfram.com/mathematica/online/

${ }^{9}$ This feature is known from software development environments (IDEs). Since Isabelle/jEdit realises this feature on terms [43], it appears an indispensable requirement for mathematics software at the state of the art.
} 
of mathematics, distinguishing it from other sciences. As, in history of science, rigorous logic was important to overcome belief and magic, comprehending this main property is an important goal for education in mathematics and in science. However, educational practice shows, that this goal is hard to achieve.

The educational gain from mechanised "models of mathematics" can be seen as follows: Many students prefer explanations in human language over looking at the structure of formulas - where the latter is indispensable for developing confidence in mathematics and for overcoming the impression of authority or magic eventually. Software works mechanically on formulas (and bypasses natural language). Thus it continuously offers direct experience with the mechanical nature of mathematics (the science of mechanisation of thinking [6]) — at any time a student is ready to recognise it, a steady mechanical offer to mature the understanding of formal language. Such understanding wouldmake academic lectures on formal logic, on meta-mathematics, on theory of science and the like better comprehensible. And, probably, freshmen would start with more confidence (and in larger numbers) into science studies.

$\boldsymbol{L I}$ features "systems that explain themselves" as already addressed in [31, 32] and increases the range of learning scenarious to be supported by software: from high-school up to elderly, who want to understand "what mathematics really is", from organised learning at various institutions up to individual study in private settings, from independent learning via learning by trial and error via "flipped classroom instruction" [9], private teaching and exercising difficult problem solutions up to written exams with automated evaluation.

The crucial feature for systems that explain themselves in mathematics is find_next_step as introduced on $\mathrm{p} 81$; a student always can request a hint from the system for a next step towards a solution and this feature also makes clear, that flexible limitation is needed to adapt to realistic learning scenarios. Such adpation is concern of a dialogue-module: by use of pattern-matching on the next step a hint can be given in full or partially (from a formula or a tactic, see the example calculation on $\mathrm{p} .82$ ), can be a list of options or can be idle (in case of a written exam).

Such a dialogue-module is implemented as a stub in ISAC's prototype [22], but several field tests [35, 33, 34] already clarified a lot of requirements for such a module; realisation shall be in collaboration with experts in educational psychology — which exhibits a great advantage of $L I$ over present principles of educational software: design and development in computer mathematics (writing $L I$-programs, mechanising deductive knowledge) are strictly separated from design and development of dialogues (extending prototypes like [18] and adopting educational theories).

\section{Adaptation of Isabelle's Functions}

Isabelle's function package presents functions in "inner syntax" to users, i.e. as terms in Isabelle/HOL. The $L I$ design recognised these terms suitable for parse trees of programs, Isabelle realised the same idea in a more general way with the function package a few years later - so migration from ISAC's programs to Isabelle's functions was surprisingly easy. The main features required were tactics, tacticals and program expressions as described below. 


\subsection{Tactics Creating Steps in Calculations}

The examples in the previous section showed how tactics in programs create steps in calculations. Tactics in programs are defined as follows ${ }^{10}$ :

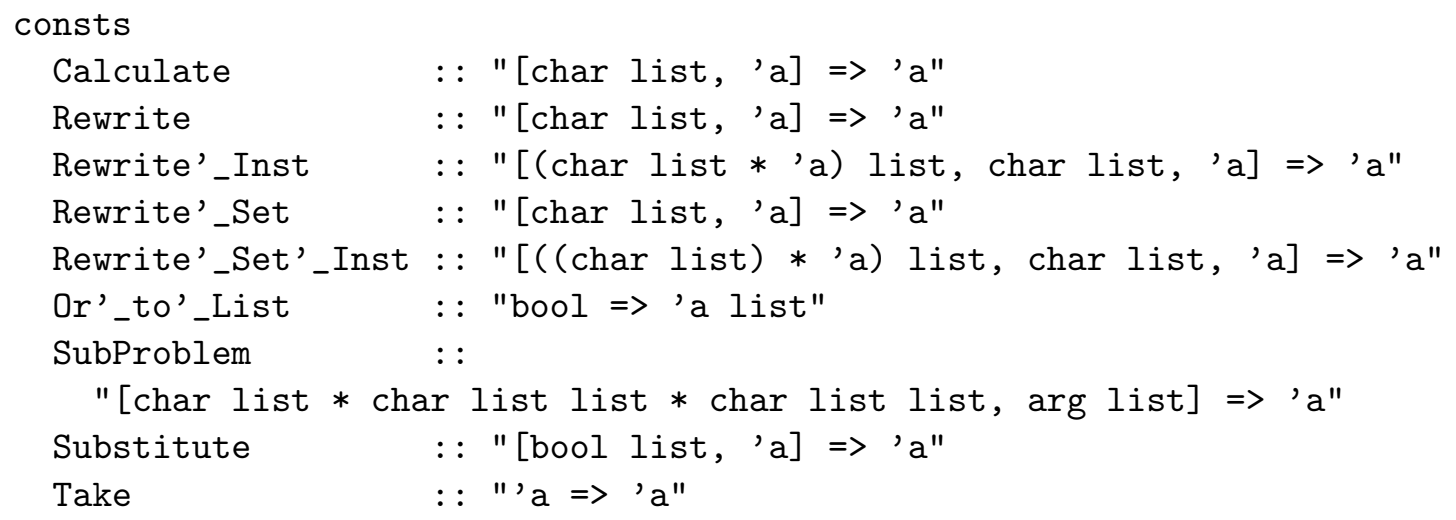

These tactics transform a term of some type 'a to a resulting term of the same type. Calculate applies operators like,,$+- *$ as char list (i.e. a string in inner syntax) to numerals of type 'a. The tactics beginning with Rewrite do exactly what they indicate by applying a theorem (in the program given by type char list) or a list of theorems, called "rule-set". The corresponding _Inst variants instantiate bound variables with respective constants before rewriting (this is due to the user requirement, that terms in calculations are close to traditional notation, which excludes $\lambda$-terms), for instance modelling bound variables in equation solving. Or_to_List is due to a similar requirement: logically appropriate for describing solution sets in equation solving are equalities connected by $\wedge, \vee$, but traditional notation uses sets (and these are still lists for convenience). SubProblems not only take arguments (arg_list like any (sub-)program, but also three references into ISAC's knowledge base (theory, formal specification, method) for guided interaction in the specification phase.

Tactics appear simple: they operate on terms adhering to one type - different types are handled by different tactics and (sub-) programs; and they cover only basic functionality - but they operate on terms, which are well tooled by Isabelle and which can contain functions evaluated as program expressions (which will be introduced in $\$ 3.3$ below).

Section $\$ 2.2$ showed how tactics can be input by students. So tactics in programs have analogies for user input with type Tactic.input defined at ${ }^{11}$. These tactics also cover the specification phase (wich is out of scope of the paper). And there is another Tactic.T defined a ${ }^{12}$ for internal use by the mathematics-engine, which already appeared in the signature of Lucas-Interpretation on $p .81$.

\subsection{Tacticals Guiding Flow of Execution}

The example on $\mathrm{p} 83$ for canonical rewriting showed, how tacticals guide the flow of execution. The complete list of tacticals is as follows.

\footnotetext{
${ }_{10}^{10}$ https://hg.risc.uni-linz.ac.at/wneuper/isa/file/df1b56b0d2a2/src/Tools/isac/ProgLang/Prog_ Tac. thy\#136

${ }^{11}$ https://hg.risc.uni-linz.ac.at/wneuper/isa/file/df1b56b0d2a2/src/Tools/isac/MathEngBasic/ tactic-def.sml\#l122

${ }^{1 /}$ https://hg.risc.uni-linz.ac.at/wneuper/isa/file/df1b56b0d2a2/src/Tools/isac/MathEngBasic/ tactic-def.sml\#1241
} 


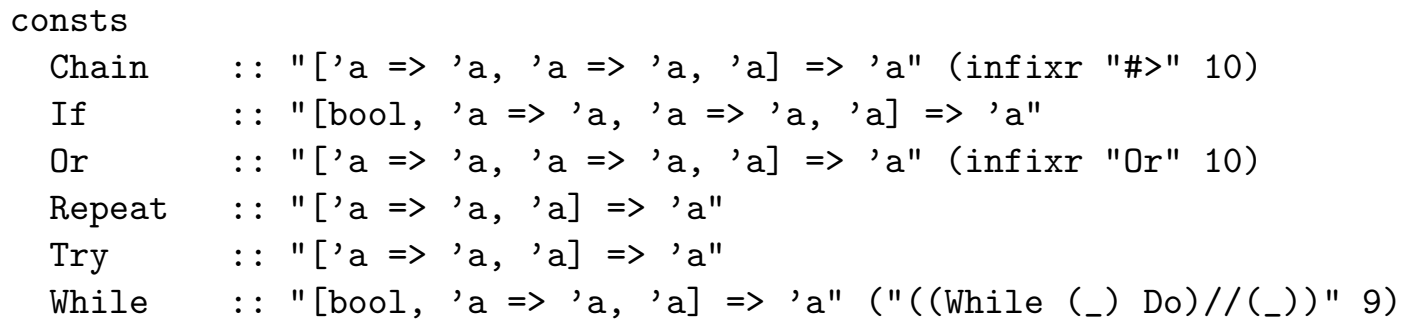

Chain is forward application of functions and made an infix operator \# $>$; If decides by boolean expression for execution of one of two arguments of type 'a Rightarrow 'a (which can be combinations of tacticals or tactics); Or decides on execution of one of the arguments depending of applicability of tactics; Repeat is a one way loop which terminates, if the argument is not applicable (e.g. applicability of a theorem for rewriting on a term) any more; Try skips the argument if not applicable and While is a zero way loop as usual.

\subsection{Program Expressions to be Evaluated}

Some tacticals, If and While, involve boolean expressions, which need to be evaluated: such expressions denote another element of programs. This kind of element has been shown in the example on $\mathrm{p} 82$ as argument of Take: sometimes it is necessary to pick parts of elements of a calculation, for instance the last element from a list. So Isabelle's List is adapted for ISAC's purposes in List $q^{13}$. Such expressions are substituted from the environment in Istate.T, evaluated to terms by rewriting (and for that purpose using the same rewrite engine as for the tactics Rewrite*) and marked by the constructor Term_Val which is introduced below.

\section{Implementation of $L I$}

The implementation of the interpreter is as experimental as is the respective programming language introduced in the previous section. So below there will be particularly such implementation details, which are required for discussing open design $\&$ implementation issues.

All of the function package's syntactic part plus semantic markup is perfect for $L I$. The evaluation part of the function package, however, implements automated evaluation in one go and automated codegeneration [12] - both goals are not compatible with ISAC's goal to feature step-wise construction of calculations, so this part had to be done from scratch.

\subsection{Scanning the Parse Tree}

Isabelle's function package parses the program body from function definitions to terms, the data structure of simply typed $\lambda$ terms, which also encode the objects of proofs. Thus there is a remarkable collection of tools, readily available in Isabelle; but this collection does not accommodate the requirement of scanning a term to a certain location, remembering the location and returning there later, as required by $L I$. So this has been introduced

datatype $\operatorname{lrd}=\mathrm{L}|\mathrm{R}| \mathrm{D}$

type path $=$ lrd list

${ }_{13}^{13}$ https://hg.risc.uni-linz.ac.at/wneuper/isa/file/df1b56b0d2a2/src/Tools/isac/ProgLang/ListC. thy 


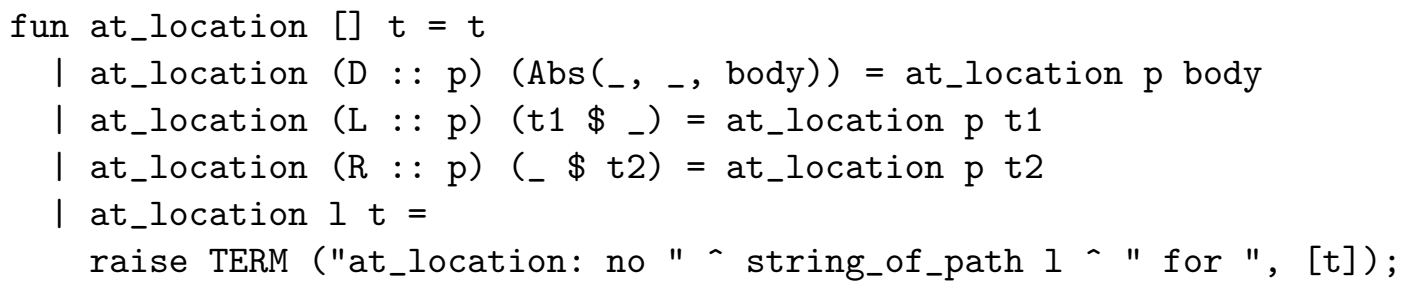

with a path to a location according to the term constructors $\$$ and Abs. This is an implementation detail; an abstract, denotational view starts with this datatype

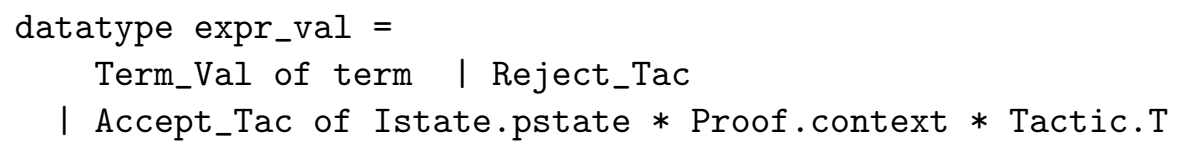

which models the meaning of an expression in the parse-tree: this is either a Term_Value (introduced in $\$ 3.3$ or a tactic (introduced in $\$ 3.1$; the latter is either accepted by Accept_Tac or rejected by Reject_Tac; an error value is still missing. The arguments of the above constant Accept_Tac are the same as introduced for $L I$ in $\$ 2.1$. Thus expr_val is the return value for functions scanning the parse-tree for an acceptable tactic:

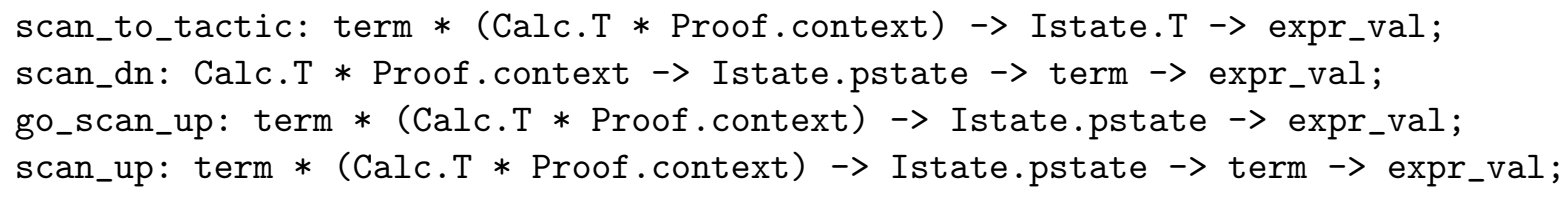

The first argument of each function above, if of type term, is the whole program required for scanning given a particular path using at_location. This is not required by scan_dn, which is simple top-down scanning. The rightmost argument, if of type term, serves matching in scan_dn and in scan_up 14

The central function is scan_to_tactic which accomplishes what the identifier indicates: if a student creates a next step at a current position in the Solution of a problem, then this position is associated with a certain path in the parse-tree, see example on $\mathrm{p} 82 . L I$ supports creating steps by one of the three actions described on $\mathrm{p} 83$. According to the action chosen $L I$ scans from the certain path to the next tactic found in the parse-tree in order to either check the input formula (1) or tactic (2), or to propose a next step (3). Function scan_to_tactic calls either scan_dn, if interpretation has just started a new program (path $=$ []) or scan_dn via go_scan_up:

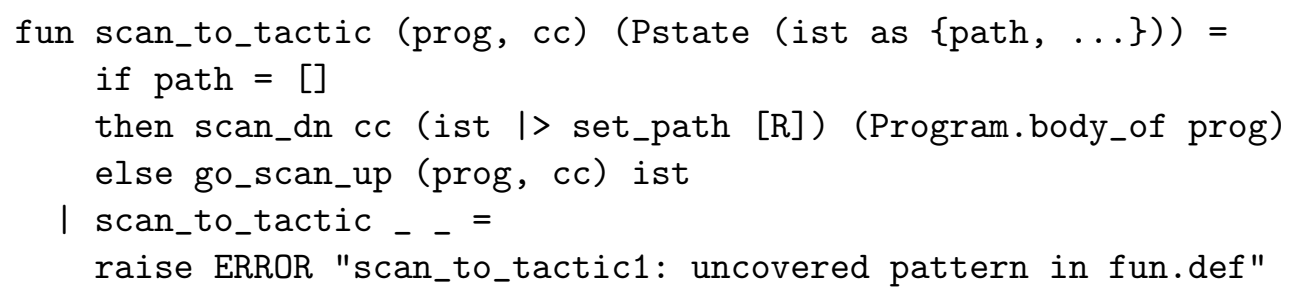

scan_dn sets the path in the interpreter state ist to R, the program body, while go_scan_up goes one level up the path in order to call scan_up. The latter uses the path found in ist, the interpreter state from the previous step.

\footnotetext{
${ }^{14}$ The code for these functions is found at https://hg.risc.uni-linz.ac.at/wneuper/isa/file/113997e55e71/ src/Tools/isac/Interpret/lucas-interpreter.sml\#l125 and at https://hg.risc.uni-linz.ac.at/wneuper/ isa/file/113997e55e71/src/Tools/isac/Interpret/lucas-interpreter.sml\#l192 respectively.
} 
It might be helpful to state, that the functions scan_dn and scan_up need not be mutually recursive: as soon as these functions have found their goal, an appropriate tactic, $L I$ terminates, stores the current state and hands over control to the student. Afterwards, depending on the path, either scan_dn or scan_up are called, so they are independent from each other.

\subsection{Use of Isabelle's Contexts}

Isabelle's "logical context represents the background that is required for formulating statements and composing proofs. It acts as a medium to produce formal content, depending on earlier material (declarations, results etc.)." [41]. The ISAC-prototype introduced Isabelle's Context in collaboration with a student [23], now uses them throughout construction of problem solutions and implements a specific structure Context 15 ,

At the beginning of the specification phase (briefly touched by tactic SubProblem in $\$ 2.2$ and explained in little more detail in the subsequent section) an Isabelle theory must be specified, this is followed by Proof_Context.init_global in the specification module. Then Proof_Context.initialise' takes a formalisation of the problem as strings, which are parsed by Syntax.read_term using the context, and finally the resulting term's types are recorded in the context by Variable.declare_constraints. The latter relieves students from type constraints for input terms. Finally, as soon as a problem type is specified, the respective preconditions are stored by ContextC.insert_assumptions.

During $L I$ the context is updated with assumptions generated by conditional rewriting and by switching to and from sub-programs. An example for the former is tactic SubProblem applied with theorem $x \neq 0 \Rightarrow\left(\frac{a}{x}=b\right)=(a=b \cdot x)$ during equation solving.

A still not completely solved issue is switching to and from SubProblems; the scope of an interpreter's environment is different from a logical context's scope. When calling a sub-program, ISAC uses Proof_Context.initialise, but returning execution from a SubProblem is not so clear. For instance, if such a sub-program determined the solutions $[x=0, x=\sqrt{2}]$, while the calling program maintains the assumption $x \neq 0$ from above, then the solution $x=0$ must be dropped, this is clear. But how determine in full generality, which context data to consider when returning execution to a calling program? Presently the decision in ContextC.subpbl_to_caller ${ }^{16}$ is, to transfer all content data which contain at least one variable of the calling program and drop them on contradiction.

\subsection{Guarding and Embedding Execution}

partial_functions as used by $L I$ are alien to HOL for fundamental reasons. And when the $I S A C$ project started with the aim to support learning mathematics as taught at engineering faculties (in that generality, see $\$ 2.3$, it was clear, that formal specifications should guard execution of programs under $L I$ (i.e. execution only starts, when the specification's preconditions evaluate to true).

So formal specification is required for technical reasons and for educational reasons in engineering education. The $I S A C$-project designed a separate specification phase, where input to a problem and corresponding output as well as preconditions and post-condition are handled explicitly by students; example in Fig 1 shows several SubProblems, which lead to mutual recursion between specification phase

\footnotetext{
${ }^{15}$ A closing "C" indicates an ISAC extension, see https://hg.risc.uni-linz.ac.at/wneuper/isa/file/ $00612574 \mathrm{cbfd} / \mathrm{src} / \mathrm{Tools} / \mathrm{isac} / \mathrm{CalcElements/contextC.sml}$

${ }^{i}$ https://hg.risc.uni-linz.ac.at/wneuper/isa/file/ce071aa3eae4/src/Tools/isac/CalcElements/ contextC.sml\#173
} 
and phases creating a solution (the latter supported by $L I$ ). Embedding $L I$ into a dialogue-module is required in order to meet user requirements as already discussed in $\$ 2.3$

\section{Lessons Learned ...}

As already mentioned, development of Isabelle and development of $L I$ went in parallel for a long time - a great opportunity for learning in the $I S A C$-project.

\section{1 ... from the Isabelle Project}

"Isabelle was not designed; it evolved. Not everyone likes this idea" said Lawrence C. Paulson in "Isabelle: The Next 700 Theorem Provers" [38].

When the ISAC-project started about the year 2000, Isabelle's code structure still reflected the enormous efforts of Paulson to make a great idea a usable product. Such a situation naturally leads to code, where placement of code is determined by the context of new feature requests. In the meanwhile Isabelle evolved to a didactic model in functional programming at a large scale: polymorphic higher order functions take complex function-arguments, which allow to postpone type definitions to locations according to functionality; and such functionality is distilled to small abstract structures. Together with canonical argument order, function combinators and canonical iteration [41, p. 15-17] this gives elegant code with almost no glue, so the hint "never copy \& paste a piece of code" could disappear from the implementation manual some time ago. And now a layered structure becomes apparent, best reflected by exception hierarchies. So Isabelle is in a state, where "everything can be changed anywhere in the code" in research\&development.

Also Isabelle's development process is a didactic model in efficient collaborative development distributed all around the world and in minimisation of administrative efforts. Visible outcomes from this process are formally checked documentation and a code repository with minimal change-sets, which denote essential feature changes with a minimum of updated code.

The ISAC-project started in a situation, where software and user requirements might have been as unclear as with early Isabelle, albeit on another level. ISAC's present code structure still looks much more like (very!) early Isabelle than present Isabelle. This paper was the occasion to make $L I$-related components in ISAC as close to Isabelle's style — and it is a hard experience, that this cannot be done in one go and will require many "rounds of reform": ISAC's code structure, even around $L I$, is still far off Isabelle's quality. This is the same with minimal change-sets: in the present state of ISAC's code so much improvements occur along current work, that it is just inefficient to separate respective change-sets in many cases.

\section{2 ... from Isabelle's Function Package}

When the development of ISAC started, a glance at Isabelle's front-end convinced everyone, that educational software could not use it. Now, two decades later, it appears clear, that ISAC is best advised to re-use Isabelle/JEdit based on Isabelle/PIDE, the integrated proof development environment as the state of the art. This is particularly evident from the work presented in this paper, shifting Lucas-Interpretation into Isabelle/Isar's function definition, which makes all the advanced features of Isabelle/JEdit available for the working programmer: 
- Syntax errors are indicated accurately at the right location; finding errors in programs represented as strings was a nightmare, if programs comprised more than a couple lines of code.

- Type annotations disappear from the program and sidestep to the heading signature; the result is much better readable.

- Syntax highlighting indicates how identifiers are interpreted, as constants, as free variable, as strings, etc - very instructive for working programmers.

- Free variables on the right-hand-side of assignments are rejected by the function package, while these were accepted by term parsing.

- Semantic annotations support the programmer, in particular the tooltip popups triggered by hovering and clicking with the mouse, see [42, p. 30].

These features came for free, when ISAC's programs were shifted into Isabelle/Isar's function definition — and were immediately fruitful: Not only the implementation of programs is much more efficient, also errors have been revealed. The features helped to detect additional free variables on the right-hand side in programs (see for instance this kind of errors in [22, p. 92]) and triggered improved handling of program arguments in $L I$.

\section{3 ... for Further ISAC-Development}

What ISAC can learn from Isabelle's code structure and from Isabelle's development process has been described in $\$ 5.1$, while Isabelle's packaging and deployment has not yet been mentioned, ISAC would benefit as well.

Future development in ISAC will follow a list of steps as announced in [22, p. 102-103]; the work on $L I$ presented in this paper is the first respective step and confirms the relevance of the other steps. However, in the meanwhile difficulties in funding a corresponding project became apparent. Indeed, the many challenges already identified in [13], suggest intermediate steps on the way to educational software for engineering disciplines as aimed at in the $I S A C$-project.

A case study on GCD [28] already investigated possibilities for such intermediate steps: the Euclidean Algorithm creates a "calculation" as presented above, if the respective invariant (or fixpoint) is output at each recursive call of the algorithm - in this example $L I$ could immediately be used to explain and to exercise calculation of the greatest common divisor of integers and polynomials. So the next challenge is to adapt $L I$ such that arbitrary functions can be executed step-wise - as an experimental approach to study algorithms in the Archive of Formal Proofs ${ }^{17}$. Algorithms in formal logic like Resolution, Binary Constraint Propagation or the DPLL algorithms can be implemented for step-wise interpretation by $L I$ already at the present state.

Such intermediate steps postpone the requirement of 2-dimensional term representation on screen - the requirement identified as most urgently missing in $I S A C$ 's field tests, and also not provided by Isabelle/jEdit: Isabelle's line-oriented presentation of terms perfectly accomplishes user requirements for proof assistants but not for mathematics in general, where fractions are read as $\frac{a}{b}$ and not as $a / b$. However, Isabelle's semantic markup in the front-end with links to types, definitions etc is indispensable in software at the actual state of computer mathematics. Presently there is no formula editor available, which features both, 2-dimensional representation and semantic markup - a separate challenge for academic open source development: just add two integers to Isabelle's Position, re-use what Knuth has implemented in $\mathrm{LTT}_{\mathrm{E}} \mathrm{X}$ for an editor (probably embedded into Isabelle/jEdit) — and create significant impact on mathematics software of various kinds (including Isabelle itself)!

\footnotetext{
${ }^{17}$ https://www.isa-afp.org/
} 


\section{Summary and Conclusions}

This paper gave the first technically concise description of Lucas-Interpretation $(L I)$ and showed how concepts from Automated Reasoning support flexible student interaction by reliable check of student input. The description focuses key points and gives many pointers into the code in a freely accessible repository ${ }^{18}$. This is to invite readers to re-use prototyped code and/or the concept of $L I$; the previous section gave some hints for re-use and for future development, more will be thinkable after further experiences.

The example from structural engineering on $\mathrm{p} 82$ makes two practical consequences of $L I$ clear. Firstly, all kinds of mathematical problems, which can be described by an algorithm, can be associated with automatically generated user-guidance using $L I$ - this covers most of mathematics as taught at arbitrary engineering faculties. Secondly implementation of respective algorithms is simple, see Fig 1 , and programming becomes more efficient with each SubProblem implemented earlier: re-using these makes development sustainable.

Final conclusions address education, since $L I$ has been invented for educational purposes and the $I S A C$-project tries to adopt and adapt technologies with pedagogical concepts in mind.

The migration of $L I$ to the function package appears to illustrate the flexible conception of the Isabelle framework, and the integrative nature of this conception confirms the hope to realise "complete, transparent \& interactive models of mathematics" [32] for education. Such mechanical models might provide experience with mathematics as the "discipline in mechanisation of thinking" (Bruno Buchberger [6]) as an indispensable complement to teaching mathematics with human intuition - an experience required to understand not only the strengths, but also the limitations of mathematical thinking technology [9].

\section{References}

[1] Archive of Formal Proofs. http://afp. sourceforge.net.

[2] S. Amani, A. Hixon, Z. Chen, C. Rizkallah, P. Chubb, L. O'Connor, J. Beeren, Y. Nagashima, J. Lim, T. Sewell, J. Tuong, G. Keller, T. Murray, G. Klein \& G. Heiser (2016): Cogent: Verifying HighAssurance File System Implementations. In: International Conference on Architectural Support for Programming Languages and Operating Systems, Springer Berlin / Heidelberg, Atlanta, GA, USA, pp. 175-188, doi: $10.1145 / 2872362.2872404$

[3] R.-J. Back (2010): Structured derivations: a unified proof style for teaching mathematics. Formal Aspects of Computing 22(5), pp. 629-661, doi 10.1007/s00165-009-0136-5.

[4] Ralph-Johan Back, Victor Bos \& Johannes Eriksson (2007): MathEdit: Tool Support for Structured Calculational Proofs. Technical Report 854, TUCS. Available at http://tucs.fi/publications/view/?pub_ id=tBaBoEr07a.

[5] F. Botana, M. Hohenwarter, P. Janičić, Z. Kovács, I. Petrović, T. Recio \& S. Weitzhofer (2015): Automated Theorem Proving in GeoGebra: Current Achievements. Journal of Automated Reasoning 55(1), pp. 39-59, doi:10.1007/s10817-015-9326-4.

[6] B. Buchberger (2013): The Role of Mathematical Thinking for 21st Century Society. Invited talk at The 2nd International Conference on Mathematics and Technology in Mathematics Education.

[7] Jasmin C.Blanchette: Hammering Away. A User's Guide to Sledgehammer for Isabelle/HOL. contained in the Isabelle distribution. Available at http://isabelle.in.tum.de/doc/sledgehammer.pdf

\footnotetext{
${ }^{18}$ https://hg.risc.uni-linz.ac.at/wneuper/isa/
} 
[8] David M. Cerna (2019): Evaluation of the VL Logic (342.208-9) 2018W End of Semester Questionnaire. RISC Report Series, Research Institute for Symbolic Computation (RISC), Johannes Kepler University Linz, Schloss Hagenberg, 4232 Hagenberg, Austria.

[9] Maurice Chiodo \& Toby Clifton (2019): The Importance of Ethics in Mathematics. Newsletter of the European Mathematical Society 114, pp. 34-37, doi 10.4171/NEWS/114/9.

[10] Gabriella Daróczy \& Walther Neuper (2013): Error-Patterns within "Next-Step-Guidance" in TP-based Educational Systems. In: eJMT, the Electronic Journal of Mathematics \& Technology, 7, pp. 175-194. Available at https://php.radford.edu/ ejmt/ContentIndex.php\#v7n2. Special Issue "TP-based Systems and Education".

[11] W. D. Farmer, J. D. Guttman \& F. J. Thayer (1993): IMPS: An Interactive Mathematical Proof System. Journal of Automated Reasoning 11(2), pp. 213-248, doi:10.1007/BF00881906.

[12] Florian Haftmann: Code generation from Isabelle/HOL theories. Contained in the Isabelle distribution. Available at http://isabelle.in.tum.de/doc/prog-prove.pdf.

[13] Florian Haftmann, Cezary Kaliszyk \& Walther Neuper (2010): CTP-based programming languages ? Considerations about an experimental design. ACM Communications in Computer Algebra 44(1/2), pp. 27-41, doi: $10.1145 / 1838599.1838621$

[14] Florian Haftmann \& Tobias Nipkow (2010): Code Generation via Higher-Order Rewrite Systems. In Matthias Blume, Naoki Kobayashi \& Germán Vidal, editors: Functional and Logic Programming, Lecture Notes in Computer Science 6009, Springer Berlin / Heidelberg, pp. 103-117, doi:10.1007/978-3-642-12251-4_9.

[15] Predrag Janičić (2006): GCLC - a tool for constructive euclidean geometry and more than that. In: Mathematical Software - ICMS 2006, 4151, pp. 58-73, doi:10.1007/11812289_22

[16] Stephen C. Johnson (1975): Yacc: Yet Another Compiler-Compiler. Technical Report 32, AT\&T Bell Laboratories, Murray Hill, New Jersey. Retrieved 31 January 2020.

[17] Stefan Karnel (2002): Größte gemeinsame Teiler in Polynomringen und Implementierung im ISAC-Projekt. Master's thesis, University of Technology, Institute of Mathematics, Graz, Austria. Available at https: //static.miraheze.org/isacwiki/3/3e/GGTs-von-Polynomen.pdf

[18] Markus Kienleitner (2012): Towards "NextStep Userguidance" in a Mechanized Math Assistant. Master's thesis, IICM, Graz University of Technology. Available at https://static.miraheze.org/isacwiki/ 0/0d/Mkienl_bakk.pdf

[19] Boris Koichu \& Alon Pinto (2019): The Seoncdary-Tertiary Transition in Mathematics. What are our current challenges and what can we do about them? EMS Newsletter, pp. 34-35, doi:10.4171/NEWS.

[20] Alexander Krauss: Defining Recursive Functions in Isabelle/HOL. Munich. Available at http:// isabelle.in.tum.de/doc/functions.pdf Part of the Isabelle distribution.

[21] Alexander Krauss (2006): Partial Recursive Functions in Higher-Order Logic. In Ulrich Furbach \& Natarajan Shankar, editors: Automated Reasoning, Third International Joint Conference, IJCAR 2006, Lecture Notes in Computer Science 4130, Springer, pp. 589-603, doi $10.1007 / 11814771 \_48$

[22] Alan Krempler \& Walther Neuper (2018): Prototyping "Systems that Explain Themselves" for Education. In Pedro Quaresma \& Walther Neuper, editors: Proceedings 6th International Workshop on Theorem proving components for Educational software, Gothenburg, Sweden, 6 Aug 2017, Electronic Proceedings in Theoretical Computer Science 267, Open Publishing Association, pp. 89-107, doi:10.4204/EPTCS.267.6.

[23] Mathias Lehnfeld (2011): Verbindung von 'Computation' und 'Deduction' im ISAC-System. Master's thesis, Institut für Computersprachen, Technische Universität Wien. Bakkalaureate project.

[24] Peter Lucas (1978): On the Formalization of Programming Languages: Early History and Main Approaches. In D. Bjørner \& C. B. Jones, editors: The Vienna Development Method: The Meta-Language, LNCS 16, Springer, doi:10.1007/3-540-08766-4_8.

[25] Roman E. Maeder (2012): Programming in Mathematica, 3rd edition. Addison-Wesley, Reading, Mass. 
[26] Walther Neuper (2001): Reactive User-Guidance by an Autonomous Engine Doing High-School Math. Ph.D. thesis, IICM - Inst. f. Softwaretechnology, Technical University, A-8010 Graz. Available at https: //static.miraheze.org/isacwiki/8/8a/Wn-diss.pdf

[27] Walther Neuper (2012): Automated Generation of User Guidance by Combining Computation and Deduction. In Pedro Quaresma \& Ralph-Johan Back, editors: Electronic Proceedings in Theoretical Computer Science, 79, Open Publishing Association, pp. 82-101, doi:10.4204/EPTCS.79.5.

[28] Walther Neuper (2014): GCD - A Case Study on Lucas-Interpretation. In: Joint Proceedings of the MathUI, OpenMath and ThEdu Workshops and Work in Progress track at CICM, Coimbra, Portugal. Available at http://ceur-ws.org/Vol-1186/paper-17.pdf. Urn:nbn:de:0074-1186-1.

[29] Walther Neuper (2016): Lucas-Interpretation from Users' Perspective. In: Joint Proceedings of the FM4M, MathUI, and ThEdu Workshops, Doctoral Program, and Work in Progress at the Conference on Intelligent Computer Mathematics, Bialystok, Poland, pp. 83-89. Available at http://cicm-conference.org/ 2016/ceur-ws/CICM2016-WIP.pdf. Urn:nbn:de:0074-1785-8.

[30] Walther Neuper (2018): Lucas-Interpretation from Programmers' Perspective. Available at https:// static.miraheze.org/isacwiki/8/83/Lucin-prog-view.pdf. Abstract for ThEdu'18.

[31] Walther Neuper (2018): Mechanical Explanation in "Systems that explain themselves". In Osman Hasan et al., editors: Workshop Papers at 11th Conference on Intelligent Computer Mathematics CICM 2018, Conference on Intelligent Computer Mathematics CICM, Hagenberg, Austria. Available at https://www. cicm-conference.org/2018/infproc/paper1.pdf Urn:nbn:de:0074-2307-7.

[32] Walther Neuper (2019): Technologies for "Complete, Transparent \& Interactive Models of Math" in Education. In Pedro Quaresma \& Walther Neuper, editors: Proceedings 7th International Workshop on Theorem proving components for Educational software, Oxford, United Kingdom, 18 july 2018, Electronic Proceedings in Theoretical Computer Science 290, Open Publishing Association, pp. 76-95, doi $10.4204 /$ EPTCS.290.6.

[33] Walther Neuper \& Christian Dürnsteiner (2007): Angewandte Mathematik und Fachtheorie mithilfe adaptierter Basis-Software. Technical Report 683, IMST - Innovationen Machen Schulen Top!, University of Klagenfurt, Institute of Instructional and School Development (IUS), 9010 Klagenfurt, Sterneckstrasse 15. Available at https://www.imst.ac.at/imst-wiki/images/f/f9/683_Kurzfassung_Neuper.pdf.

[34] Walther Neuper, Johannes Reitinger \& Angelika Gründlinger (2008): Begreifen und Mechanisieren beim Algebra Einstieg. Technical Report 1063, IMST - Innovationen Machen Schulen Top!, University of Klagenfurt, Institute of Instructional and School Development (IUS), 9010 Klagenfurt, Sterneckstrasse 15. Available at https://www.imst.ac.at/imst-wiki/images/9/9d/1063_Langfassung_Reitinger.pdf.

[35] Walther Neuper et al. (2006): Angewandte Mathematik und Fachtheorie. Technical Report 357, IMST - Innovationen Machen Schulen Top!, University of Klagenfurt, Institute of Instructional and School Development (IUS), 9010 Klagenfurt, Sterneckstrasse 15. Available at http://imst.uni-klu.ac.at/imst-wiki/ index.php/Angewandte_Mathematik_und_Fachtheorie.

[36] Tobias Nipkow: Programming and Proving in Isabelle/HOL. contained in the Isabelle distribution. Available at http://isabelle.in.tum.de/doc/prog-prove.pdf.

[37] Tobias Nipkow, Lawrence C. Paulson \& Markus Wenzel (2008): Isabelle/HOL, a proof assistant for highorder logic. Springer Verlag.

[38] Lawrence C. Paulson (1990): Isabelle: The Next 700 Theorem Provers. In P. Odifreddi, editor: Logic and Computer Science, Academic Press, pp. 361-386. Available at https://arxiv.org/abs/cs/9301106

[39] Pedro Quaresma \& Ralph-Johan Back, editors (2012): Proceedings First Workshop on CTP Components for Educational Software (THedu'11). 79, Open Publishing Association, doi:10.4204/EPTCS.79.

[40] Y. Shi, Y. Ma, J. MacLeod et al. (2019): College students' cognitive learning outcomes in flipped classroom instruction: a meta-analysis of the empirical literature. Journal of Computers in Education, pp. 1-25, doi $10.1007 / \mathrm{s} 40692-019-00142-8$. 
[41] Makarius Wenzel: The Isabelle/Isar Implementation. Contained in the Isabelle distribution. Available at http://isabelle.in.tum.de/doc/implementation.pdf

[42] Makarius Wenzel: Isabelle/jEdit. Munich. Available at http://isabelle.in.tum.de/doc/jedit.pdf Part of the Isabelle distribution.

[43] Makarius Wenzel (2014): System description: Isabelle/jEdit in 2014. In: Proceedings Eleventh Workshop on User Interfaces for Theorem Provers, UITP 2014, Vienna, Austria, 17th July 2014., pp. 84-94, doi $10.4204 /$ EPTCS.167.10. 